\title{
ON A GENERALIZED VOLTERRA EQUATION IN HILBERT SPACE ${ }^{1}$
}

\author{
ROMANO M. DE SANTIS
}

\begin{abstract}
The objective of this paper is to establish a fundamental theorem on the existence and uniqueness of the solution of a generalized Volterra equation in Hilbert space. Some applications to the areas of abstract functional analysis and modern mathematical systems theory are pointed out.
\end{abstract}

1. Introduction. A bounded linear operator $P$ on a Hilbert space $H$ is called an orthoprojector if for all pairs $x, y \in H^{2}$ one has $\langle P x, y\rangle=\langle x, P y\rangle$, and $P^{2} x=P x$. If $P_{1}$ and $P_{2}$ are two orthoprojectors, the symbol $P_{1}<P_{2}$ is used to indicate that $P_{1} H$ (the range of $P_{1}$ ) is contained in $P_{2} H$. A set $\mathfrak{B}$ of orthoprojectors is called a chain if for every pair $P_{1}, P_{2} \in \mathfrak{B}$ one has either $P_{1}<P_{2}$ or $P_{2}<P_{1}$; the chain $\mathfrak{B}$ is called bordered if it contains the null operator 0 , and the identity operator $I ; \mathfrak{B}$ is closed if it has the property that whenever a sequence of orthoprojectors $\left\{P_{i}\right\} \in \mathfrak{B}$ is such that $\lim \left\{P_{i} x\right\}=P x$ for all $x \in H$, then $P \in \mathfrak{B}$. A chain $z$ is a partition of $\mathfrak{B}$ if $z$ is composed of a finite number of orthoprojectors in $\mathfrak{B}$. If $z_{1}$ and $z_{2}$ are two partitions of $\mathfrak{B}$, the symbol $z_{2} \supset z_{1}$ will indicate that if $P \in z_{1}$, then $P \in z_{2}$.

Suppose that $\Phi(\cdot)$ is an operator valued function which associates to each partition $z$ of $\mathfrak{B}$ a bounded operator $\Phi(z): H \rightarrow H$. The function $\Phi(\cdot)$ is said to have as limit in the norm the operator $T$, if given any $\varepsilon>0$ it is possible to find a partition $z_{\varepsilon}$ of $\mathfrak{B}$ such that for every other partition $z$ with the property $z \supset z_{\varepsilon}$, one has $|\Phi(z)-T|<\varepsilon$.

Suppose that $Y$ is a bounded operator in $H$, where $H$ is equipped with a bordered and closed chain $\mathfrak{B}$, and consider the operator valued function $S(\cdot)$ defined as follows: if $z=\left\{0=P_{0}<P_{1} \cdots<P_{n-1}<P_{n}=I\right\}$ is a partition of the bordered and closed chain $\mathfrak{B}$, then $S(z)=\sum_{i=1}^{n} \Delta P_{i} Y P_{i-1}$, where $\Delta P_{i}=P_{i}-P_{i-1}$. If $S(\cdot)$ has as its limit in norm some operator $T$, then it will be said that the integral $(m) \int_{\mathfrak{B}} d P Y P$ converges and equals $T$. The integrals $(m) \int_{\mathfrak{B}} P Y d P$, and $\int_{\mathfrak{B}} d P Y d P$ are defined analogously. ${ }^{2}$

Received by the editors June 6, 1972 and, in revised form, July 5, 1972.

AMS (MOS) subject classifications (1970). Primary 47H10, 47H15; Secondary 45D05, $46 \mathrm{E} 40$.

${ }^{1}$ This research was supported in part by the US Grant AFOSR 73-2427 and by the NRC Grant A8244.

${ }^{2}$ All the concepts presented up to this point are taken directly from the book of Gohberg and Kreĭn [5, $\S \S 1.3$ and 1.4] and are included here to make the paper selfcontained.

(c) American Mathematical Society 1973 
Our purpose is to state, prove and discuss the following theorem.

THEOREM 1. Let the Hilbert space $H$ be equipped with the bordered and closed chain of orthoprojectors $\mathfrak{B}$, and suppose that $X$ is a linear bounded operator such that $X=(m) \int_{\mathfrak{B}} d P X P$, and $Y$ is a bounded and Lipschitz continuous operator such that $P Y=Y P$ and $(I-P) Y=Y(I-P)$ for all $P \in \mathfrak{B}$. Then the inverse operator $(I-X Y)^{-1}$ exists, is bounded and continuous and it has the property that $P(I-X Y)^{-1}=P(I-X Y)^{-1} P$ for all $P \in \mathfrak{B}$.

To help illustrate the statement of the theorem, and the notational machinery which goes with it, it seems appropriate to conclude the section with the following simple example.

EXAMPLE. Let $H$ be given by $L_{2}(0,+\infty)$, the Hilbert space of real valued Lebesgue square integrable functions defined on the interval $(0,+\infty)$, and suppose that the operators $Y$ and $X$ are defined as follows: if $x, y, w, z \in L_{2}(0,+\infty)$, and $y=Y x, z=X w$, then:

$-y(t)=N_{l}(x(t))$, where $N_{l}(\cdot)$ is any bounded and Lipschitz continuous real valued function defined on the real line;

$-z(t)=h(t)\left[\sum_{n=0}^{\infty} g_{n} w\left(t-\Delta t_{n}\right)\right], \quad$ where $h(\cdot) \in L_{\infty}(0, \infty), \quad$ and $\lim _{t \rightarrow \infty}$ ess sup $|h(t)|=0, \Delta t_{0}>0, \Delta t_{n-1}<\Delta t_{n}$, and $\left\{g_{n}\right\}$ is a summable sequence of real numbers $\left(\sum_{n=0}^{\infty}\left|g_{n}\right|<+\infty\right)$.

In $L_{2}(0,+\infty)$ we can consider the bordered and closed chain of orthoprojectors, $\mathfrak{B}$, given by the family of truncation operators $\left\{P^{t}\right\}, t \in[0,+\infty]$, defined as follows: if $x, y \in L_{2}(0,+\infty)$ and $x=P^{t} y$, then $x(s)=y(s)$ for $s \in(0, t)$, and $x(s)=0$ for $s \in(t,+\infty)$; when $t=\infty$ then $P^{\infty} x=x$. Note that $Y$ is a bounded and Lipschitz continuous (not necessarily linear) operator, and $X$ is linear and bounded (not necessarily compact); moreover, it is not difficult to recognize that $Y$ has the property that $P Y=Y P$ and $(I-P) Y=$ $Y(I-P)$ for each $P \in \mathfrak{B}$, while $X$ is such that $X=(m) \int_{\mathfrak{B}} d P X P$. Clearly then, all the hypotheses of the theorem are satisfied, and one can conclude that the operator $(I-X Y)^{-1}$ is well defined, bounded and continuous, and it enjoys the property that $P^{t}(I-X Y)^{-1}=P^{t}(I-X Y)^{-1} P^{t}$ for every $t \in$ $[0,+\infty]$.

2. Applications. An example of application of Theorem 1 to the area of abstract functional analysis is given by the following corollary.

COROLlaRy 1. Let the Hilbert space $H$ be equipped with the bordered and closed chain of orthoprojectors $\mathfrak{B}$, and suppose that $X$ is a linear and bounded operator for which the integral $Y=(m) \int_{\mathfrak{P}} P X d P$ converges. Then for every scalar $\alpha$, the operator $(I-\alpha Y)^{-1}$ exists and it is bounded and continuous. 
Proof. From $Y=(m) \int_{\mathfrak{B}} d P X P$ it follows $\int_{\mathfrak{B}} d P Y d P=0$, and $(I-P) Y=$ $(I-P) Y(I-P)$. This leads to $\int_{\mathfrak{B}^{\prime}} d P^{\prime} Y d P^{\prime}=0$, and $P^{\prime} Y=P^{\prime} Y P^{\prime}$ for all $P^{\prime} \in \mathfrak{B}^{\prime}$, where $\mathfrak{B}^{\prime}$ is the chain of orthoprojectors defined as follows: if $P \in \mathfrak{B}$ then $I-P=P^{\prime} \in \mathfrak{B}^{\prime}$. These two properties of $Y$ are equivalent to $Y=$ $(m) \int_{\mathfrak{B}} d P^{\prime} Y P^{\prime}$, and the desired result is an immediate consequence of Theorem 1.

Observe that Corollary 1 gives a generalization of previous theorems due to Brodskii and to Gohberg and Kreinn. In particular this result was first obtained by Gohberg and Krein in the case where $X$ is a Hilbert Schmidt operator. Brodskii [1] extended its validity to the case where $X$ is linear and completely continuous and the $\mathfrak{B}$ is maximal. ${ }^{3}$ Gohberg and Kreǐn further improved Brodskiřs result by lifting the requirement about the maximality of $\mathfrak{B}$ [5, Theorem $6.1 \mathrm{~b}, \mathrm{p} .27$ ].

To appreciate some systems theory implications of Theorem 1, note that the study of the inverse operator $(I-K)^{-1}$ is equivalent to the study of the closed loop feedback system illustrated in Figure 1. A linear operator

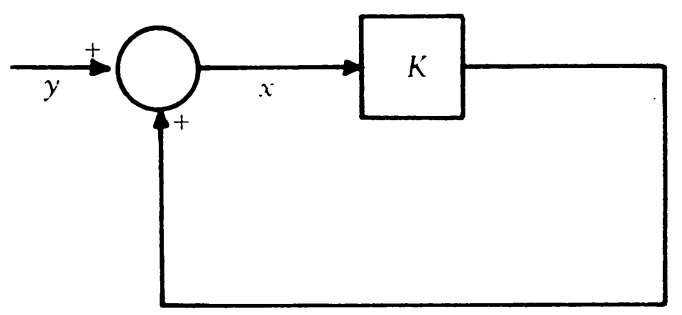

FIGURE 1

$X$ such that $X=(m) \int_{\mathfrak{B}} d P X P$ is referred to as strictly causal, and an operator $Y$ satisfying the relation $P Y=Y P$ and $(I-P) Y=Y(I-P)$ for all $P \in \mathfrak{B}$ is called memoryless (see for example [6]). According to a popular criterion of stability of a feedback system, see for example [2], Theorem 1 can now be rephrased as follows.

Corollary 2. A sufficient condition for the closed loop feedback system in Figure 1 to be stable is that $K$ is given by the composition of a memoryless and Lipschitz continuous system with a linear bounded and strictly causal system.

It is of interest to note that the systems considered in the above corollaries are somewhat similar to those which are considered, for example,

\footnotetext{
${ }^{3} \mathrm{~A}$ chain $\mathfrak{B}$ is maximal if there exists no other chain $\mathfrak{B}^{\prime}$ such that $\mathfrak{B} \subset \mathfrak{B}^{\prime}$.
} 
in the celebrated Popov Stability Criteria. For a discussion about the relationship between results of this latter type and those of the present development the reader is referred to [3] and [4].

3. Some preliminary results. To prove Theorem 1 it is helpful to recall the following lemmas. In stating these lemmas, the symbols $X, Y$, and $K$ indicate bounded operators on a Hilbert space $H$, where $H$ is assumed to be equipped with the bordered and closed chain of orthoprojectors $\mathfrak{B}$.

LemMa 1. Necessary and sufficient conditions for $X$ to be such that $X=(m) \int_{\mathfrak{B}} d P X P$, are that $\int_{\mathfrak{B}} d P X d P=0$, and $P X=P X P$ for all $P \in \mathfrak{B}$.

Lemma 2. If $X$ is such that $X=(m) \int_{\mathfrak{B}} d P X P$, and $Y$ is such that $P Y=$ $P Y P$ for all $P \in \mathfrak{B}$, then $X Y$ is such that $X Y=(m) \int_{\mathfrak{B}} d P X Y P$.

LEMma 3. If $Y$ is such that $P Y=Y P$ and $(I-P) Y=Y(I-P)$ then for every pair $P_{1}, P_{2} \in \mathfrak{B}$ one has $\left(P_{2}-P_{1}\right) Y=\left(P_{2}-P_{1}\right) Y\left(P_{2}-P_{1}\right)$.

LemMa 4. If $K$ is Lipschitz continuous and has a Lipschitz norm less than 1 , then the inverse $(I-K)^{-1}$ exists and it is bounded and continuous. Moreover, if $P K=P K P$ for all $P \in \mathfrak{B}$, then one also has $P(I-K)^{-1}=$ $P(I-K)^{-1} P$.

The details of the proofs of Lemmas 1, 2, and 3 can be found in [3], and are omitted for brevity. It suffices here to say that Lemmas 1 and 2 follow from the definition of integral, and Lemma 3 is a consequence of the fact that if $P Y=Y P$ and $(I-P) Y=Y(I-P)$, then $P Y=P Y P$ and $(I-P) Y=$ $(I-P) Y(I-P)$. In regard to Lemma 4 , a sketch of its proof is given in the Appendix.

4. Outline of the proof. Let us pose $K=X Y$ and let us start to show that the inverse operator $(I-K)^{-1}$ exists, that is: for every $y \in H$ the equation

$$
y=x-K x
$$

has a unique solution $x \in H$. Noting that $P Y=P Y P$, and applying Lemmas 1 and 2 one has

$$
P K=P K P, \text { for all } P \in \mathfrak{B},
$$

and

$$
\int_{\mathfrak{B}} d P K d P=0
$$


This latter equation implies that there exists a partition $z$ of $\mathfrak{B}, z=\{0=$ $\left.P_{0}, P_{1}, \cdots, P_{N}=I\right\}$, such that

$$
|\Delta P(i) K \Delta P(i)|<1,
$$

where $i=1,2, \cdots, N$, and $\Delta P(i)=P_{i}-P_{i-1}$.

Observe now that, by (2), the problem of finding a solution to (1) is equivalent to finding an $x \in H$ such that the following equations are satisfied

$$
\Delta P(i) y=\Delta P(i) x-\Delta P(i) K P_{i} x
$$

where $i=1,2, \cdots, N$. In the case of $i=1,(5)$ becomes

$$
\Delta P(1) y=\Delta P(1) x-\Delta P(1) K \Delta P(1) x,
$$

where, by (4), the operator $\Delta P(1) K \Delta P(1)$ is Lipschitz continuous, and has a Lipschitz norm less than 1. From Lemma 4, there exists then a welldefined and bounded operator $T^{1}: \Delta P(1) H \rightarrow \Delta P(1) H$, such that the element

$$
\Delta P(1) x=T^{1} \Delta P(1) y
$$

is the unique solution to (6) in $\Delta P(1) H$. Similarly, for $i=2$, (5) becomes

$$
\Delta P(2) y=\Delta P(2) x-\Delta P(2) K P_{2} x .
$$

Using the linearity of $X$ and Lemmas 1 and 3, this latter equation can be rewritten as follows

$$
\Delta P(2) y+\Delta P(2) K \Delta P(1) x=\Delta P(2) x-\Delta P(2) K \Delta P(2) x
$$

where, by (4), $\Delta P(2) K \Delta P(2)$ is Lipschitz continuous with Lipschitz norm less than 1, and $\Delta P(1) x$ is given by (7). Lemma 4 can then be applied again and there exists a bounded continuous operator $T^{2}: \Delta P(2) H \rightarrow \Delta P(2) H$ such that, posing

$$
\Delta P(2) x=T^{2} \Delta P(2)[y+K \Delta P(1) x],
$$

one obtains the unique solution to (8), $P_{2} x=\Delta P(1) x+\Delta P(2) x$. Proceeding by induction, suppose that, for $j=1,2, \cdots, i-1,(5)$ has the unique solutions $P_{j} x=P_{j-1} x+\Delta P(j) x$. Then, for $j=i$, (5) has the unique solution $P_{i} x=P_{i-1} x+\Delta P(i) x$, where

$$
\Delta P(i) x=T^{i} \Delta P(i)\left[y+K P_{i-1} x\right]
$$

and $T^{i}: \Delta P(i) H \rightarrow \Delta P(i) H$ is a well-defined bounded and continuous operator. This recursive relation defines the element $x=\sum_{i=1}^{N} \Delta P(i) x$, and this element is the unique solution to (1). 
To prove that $(I-K)^{-1}$ is bounded, suppose that $x, y \in H$ and $x=$ $(1-K)^{-1} y$. Observe that, for each $i=1,2, \cdots, N, \Delta P(i) x=\Delta P(i)(I-K)^{-1} y$ is defined by (11). Moreover, using the boundedness of $K$ and applying Lemma 4, it follows that there exists a set of positive real numbers $M_{1}$, $M_{2}^{\prime}, M_{2}^{\prime \prime}, M_{3}^{\prime}, M_{3}^{\prime \prime}, \cdots, M_{i}^{\prime}, M_{i}^{\prime \prime}$, such that

$$
\left|\Delta P(1)(I-K)^{-1} y\right| \leqq M_{1}|y|
$$

and for $i=2,3, \cdots, N$,

$$
\left|\Delta P(i)(I-K)^{-1} y\right| \leqq M_{i}^{\prime}|y|+M_{i}^{\prime \prime}\left|P_{i-1} x\right| .
$$

From these equations it follows that there exists a positive $M$ such that

$$
|x|=\left|(I-K)^{-1} y\right| \leqq M|y| \text {. }
$$

In regard to the continuity of $(I-K)^{-1}$, this continuity is equivalent to that of the operators $\Delta P(i)(I-K)^{-1}, i=1,2, \cdots, N$. On the other hand, $\Delta P(i)(I-K)^{-1}$ can be computed using (11) and therefore, in view of the continuity of $T^{i}$, it is continuous if $P_{i-1} x$ depends continuously on $y$. But, from (7), $P_{1} x=\Delta P(1) x$ does depend continuously on $y$. Similarly from (10), $P_{2} x=\Delta P(2) x+\Delta P(1) x$ depends continuously on $y$. By induction and (11) it follows that $P_{i-1}$ depends continuously on $y$ for each $i=2,3, \cdots, N$. It remains to show that, for each $P \in \mathfrak{B}$, one has

$$
P(I-K)^{-1} y=P(I-K)^{-1} P y .
$$

To this purpose, consider the partition $z^{\prime}=z \cup\{0, P, I\}=\left\{0=P_{0}, P_{1}, \cdots\right.$, $\left.P_{i-1}, P, P_{i}, \cdots, P_{. V}=I\right\}$. Using the notations

$$
x^{1}=(I-K)^{-1} y^{-1}, \quad x^{2}=(I-K)^{-1} y^{2},
$$

where $y^{1}=y$, and $y^{2}=P y$, from (11) one has

$$
\begin{aligned}
x^{a}=(I-K)^{-1} y^{q}= & \sum_{j=1, j \neq i}^{N} T_{j}\left[\Delta P(j)\left(y^{a}+K P_{j-1} x^{q}\right)\right] \\
& +T_{i}^{\prime}\left[\left(P-P_{i-1}\right) y^{q}+K P_{i-1} x^{q}\right] \\
& +T_{i}^{\prime \prime}\left[\left(P_{i}-P\right) y^{q}+K P x^{q}\right],
\end{aligned}
$$

where $q=1,2$. By direct inspection, it follows

$$
P_{1} x^{1}=P_{1} x^{2}, \quad P_{2} x^{1}=P_{2} x^{2}, \cdots, P_{i-1} x^{1}=P_{i-1} x^{2}, \quad P x_{1}=P x_{2} .
$$

This implies the validity of (11).

5. Appendix. In what follows we give a sketch of the proof of Lemma 4. To this purpose, we prove first that the inverse of $(I-K)$ exists. To do this it is clearly sufficient to show that, for every $y \in H$, the equation

$$
y=x-K x
$$


has one and only one solution $x \in H$. This, in turn, is equivalent to showing the existence and uniqueness of a fixed point for the operator $T_{y}: H \rightarrow H$, defined by the relation $T_{y}(x)=y+K x$. But $T_{y}$ is a contraction operator because for any pair $x_{1}, x_{2} \in H$ one has

$$
\left|T_{y}\left(x_{1}\right)-T_{y}\left(x_{2}\right)\right|=\left|K x_{1}-K x_{2}\right| \leqq l\left|x_{1}-x_{2}\right|,
$$

where $l<1$ is the Lipschitz norm of $K$. The desired result follows then by the Banach contraction mapping theorem. In regard to the boundedness and continuity of $(I-K)^{-1}$, note that $(I-K)^{-1} 0=0$, and it will be then sufficient to prove that $(I-K)^{-1}$ is Lipschitz continuous. To this purpose, observe that for any pair $x_{1}, x_{2} \in H$ one has

$$
\begin{aligned}
\left|x_{1}-K x_{1}-\left(x_{2}-K x_{2}\right)\right| & \\
& \geqq\left|x_{1}-x_{2}\right|-\left|K x_{2}-K x_{1}\right| \geqq(1-l)\left|x_{1}-x_{2}\right|
\end{aligned}
$$

where $l<1$ is once again the Lipschitz norm of $K$. Using the fact that $(I-K)$ is invertible, and posing $y_{1}=(I-K) x_{1}$, and $y_{2}=(I-K) x_{2}$, (A.2) implies that for every pair $y_{1}, y_{2} \in H$ one has

$$
\left|y_{1}-y_{2}\right| \geqq(1-l)\left|(I-K)^{-1} y_{1}-(I-K)^{-1} y_{2}\right|,
$$

that is,

$$
\left|(I-K)^{-1} y_{1}-(I-K)^{-1} y_{2}\right| \leqq(1-l)^{-1}\left|y_{1}-y_{2}\right| .
$$

To complete the proof of Lemma 4 , it remains to be checked that if one has $P K=P K P$ for $P \in \mathfrak{B}$, then one also has $P(I-K)^{-1}=P(I-K)^{-1} P$. This is clearly equivalent to showing that if $y_{1}, y_{2} \in H$ and $P y_{1}=P y_{2}$ then

$$
P(I-K)^{-1} y_{1}=P(I-K)^{-1} y_{2} \text {. }
$$

Posing $x_{1}=(I-K)^{-1} y_{1}$, and $x_{2}=(I-K)^{-1} y_{2}$, and applying the hypotheses that $P K=P K P$, one has

$$
P y_{1}=P x_{1}-P K P x_{1}, \quad \text { and } P y_{2}=P x_{2}-P K P x_{2} .
$$

Since $P y_{1}=P y_{2}$, these equalities lead to

$$
\left|P x_{1}-P x_{2}\right|=\left|P K P x_{2}-P K P x_{1}\right|
$$

and, by the hypotheses that the Lipschitz norm of $K$ is less than 1, one can conclude that $P x_{1}=P x_{2}$, and therefore that (A.3) is true.

\section{REFERENCES}

1. M. S. Brodskil̆, On the triangular representation of completely continuous operators with one-point spectra, Uspehi Mat. Nauk 16 (1961), no. 1 (97), 135-141 ; English transl., Amer. Math. Soc. Transl. (2) 47 (1965), 59-65. MR 24 \#A426. 
2. M. Damborg and A. Naylor, The fundamental structure of input-output stability for feedback systems, IEEE Trans. on System Science and Cybernetics, April, 1970.

3. R. M. De Santis, Causality structure of engineering systems, Ph.D. Thesis, University of Michigan, Ann Arbor, Mich., 1971.

4. - Causality, strict causality and invertibility for systems in Hilbert resolution spaces, Technical Report DA-AN-72-006, École Polytechnique, Montréal, Canada.

5. I. C. Gohberg and M. G. Krein, Theory and applications of Volterra operators in Hilbert space, "Nauka", Moscow, 1967; English transl., Transl. Math. Monographs, vol. 24, Amer. Math. Soc., Providence, R.I., 1970. MR 36 \#2007.

6. R. Saeks, Causality in Hilbert space, SIAM Rev. 12 (1970), 357-383.

Division d'Automatique, Département de Génie Électrique, École PolyTeChnique, Montréal, Québec, Canada 